Revista Brasileira de Odontologia Legal - RBOL

\title{
Odontología Legal
}

\section{REGLAMENTACIÓN DEL EJERCICIO PROFESIONAL EN MEDICINA Y ODONTOLOGÍA EN PARAGUAY: UNA NECESIDAD DE SALUD PÚBLICA.}

\section{Regulation of the health care and the dental profession in Paraguay: a public health necessity.}

\author{
Tamara Soledad Frontanilla RECALDE ${ }^{1,2}$, Santiago Gómez ABENTE ${ }^{2,3}$. \\ 1. Especialista en Odontologia Legal - Facultad de Odontología de Ribeirão Preto - Universidad de São Paulo. Alumna \\ de Maestría - Universidad de São Paulo. \\ 2. Socio Director del estudio PRACTICE - Asesoría legal médico-odontológica. Asunción, Paraguay. \\ 3. Abogado. Estudio Codas. Law Clerk - DLA Piper LLP - Washington D.C., Estados Unidos. Máster en Derecho \\ (LL.M.) - Universidad de Columbia en la ciudad de New York.
}

\author{
Informação sobre o manuscrito \\ Recebido em: 11 Setembro 2018 \\ Aceito em: 27 Setembro 2018
}

\author{
Autor para contato: \\ Tamara S. F. Recalde \\ Avenida Santa Luzia, 440 \\ Ribeirão Preto, São Paulo, Brasil \\ CEP: 14025-090 \\ E-mail: tfronta@gmail.com.
}

\begin{abstract}
RESUMEN
Una de las principales funciones del Estado es velar por la salud pública de sus habitantes. El Estado paraguayo desempeña esta función a través del Ministerio de Salud Pública y Bienestar Social. La mayoría de las reglamentaciones existentes en salud, fueron creadas hace varios años, encontrándose actualmente desactualizadas. Los errores en el área de la salud pueden y a menudo suelen tener como consecuencia la muerte de una persona. Es por esto que la modernización y actualización de las regulaciones del ejercicio profesional en el sector de la salud se traducen en una prioridad y una necesidad de salud pública. El objetivo de este trabajo fue exponer las problemáticas actuales existentes causadas por la falta de reglamentaciones y normativas adecuadas en el área de la salud, así como demostrar que esta reglamentación constituye una necesidad de salud pública. Fue realizada una investigación exhaustiva de las legislaciones y regulaciones locales existentes referentes al ejercicio profesional. Posteriormente, cada regulación fue analizada minuciosamente, y se procedió a una discusión sobre la aplicabilidad y las limitaciones de estas normativas para cada situación específica. A través de este trabajo, fue posible exponer la falta de regulación adecuada en el área de la salud en Paraguay, y las consecuencias negativas de esta situación, así como a concluir que la creación de un marco regulatorio actualizado y moderno es una necesidad de salud pública que promoverá los intereses de la sociedad al favorecer y facilitar la prestación de servicios de atención médica de alta calidad.
\end{abstract}

\section{PALABRAS CLAVES}

Práctica professional; Salud pública; Legislación; Odontología Legal.

\section{INTRODUCCIÓN}

Se considera profesional a la persona física que ejerce una determinada profesión, que consiste en una actividad de carácter intelectual ${ }^{1}$. Ejercicio profesional, es toda actividad técnica, científica y/o docente y su consiguiente responsabilidad; sean éstas realizadas en forma pública o privada, libremente 0 en relación de dependencia y que requieran la 
capacitación que otorga el título proporcionado por universidades oficiales o privadas reconocidas por el Estado del marco de incumbencia, fijadas a su vez por las autoridades competentes ${ }^{2}$.

Una de las principales funciones del Estado es velar por la salud pública de sus habitantes. El Estado paraguayo desempeña esta función a través del Ministerio de Salud Pública y Bienestar Social (MSPBS), órgano competente para la prestación, regulación y supervisión de todos los servicios sanitarios en el territorio nacional ${ }^{3}$.

EI MSPBS se rige por un conjunto de normas jurídicas y reglamentarias que regulan su actividad, con el objetivo de proteger los derechos, facultades $y$ atribuciones de cada sector de la salud, y a la vez garantizar la prestación eficiente de los servicios sanitarios existentes y los que se incorporen en el futuro ${ }^{3,4}$. Entre estos servicios se encuentra el ejercicio de los profesionales del sector de la salud. Las principales disposiciones normativas que regulan el actuar del MSPBS son la Constitución Nacional de la República del Paraguay de $1992^{5}$, el Código Sanitario, del $1980^{6}$ y la Ley № $1.032 / 96^{7}$ que crea el Sistema Nacional de Salud, así como los decretos que reglamentan estas leyes y las diversas resoluciones propias del Ministerio $^{2-8}$.

Cabe destacar que la mayoría de estas reglamentaciones fueron creadas hace varios años, mientras que la medicina ha avanzado -y continúa haciéndolo- a pasos agigantados. Los avances tecnológicos, el desarrollo de nuevas técnicas y las necesidades actuales de los pacientes son completamente distintas a las que estaban vigentes cuando fueron creadas estas estas regulaciones ${ }^{9}$.

Además, el marco regulatorio de la salud en Paraguay apenas se detiene a tratar la cuestión del ejercicio profesional del sector. A modo de ejemplo, para poder ejercer las diversas profesiones relacionadas a la salud, únicamente se requiere el título de una universidad habilitada y la inscripción en el MSPBS. A partir de ahí, cada profesional es libre de ejercer su actividad en la forma que crea conveniente y no existe un órgano específico que se dedique específicamente a monitorear y controlar que los profesionales de la salud actúen conforme a las mejores prácticas de la profesión, y que, si así no lo hicieren, aplique una sanción adecuada $^{10}$.

Es importante que las autoridades entiendan que un error en el área de la salud puede tener como consecuencia la muerte de una persona. Profesionales no preparados, realizando procedimientos para los cuales no fueron habilitados o que no cuentan con la formación académica necesaria, normalmente acarrean complicaciones, secuelas permanentes 0 incluso muertes que podrían haberse evitado con una mejor preparación profesional. Es por esto que la reglamentación del ejercicio profesional en el área de la salud se traduce en una prioridad y una necesidad de salud pública.

El objetivo de este trabajo es exponer las problemáticas actuales existentes debido a la falta de reglamentaciones y normativas legales en el área de la salud, así como demostrar que 
esta reglamentación constituye una necesidad de salud pública.

\section{METODOLOGÍA}

Fue realizado un análisis comparativo entre las legislaciones locales existentes referentes al ejercicio profesional, profesiones en salud, reglamentaciones y normativas legales vigentes. Posteriormente fueron analizadas cuidadosamente cada una de ellas, llevando en consideración el principio de la jerarquía y abierta una discusión sobre las problemáticas y situaciones actuales, tanto de la aplicabilidad, cuanto de las limitaciones de estas normativas en cada situación específica.

\section{RESULTADOS}

Fueron encontradas y analizadas las legislaciones locales existentes referentes al ejercicio profesional, entre ellas; la Constitución Nacional de la República del Paraguay de 1992, el Código Sanitario de 1980, la Ley no 1.032/96 que crea el Sistema Nacional de Salud, además de los decretos que reglamentan estas leyes, y diversas resoluciones propias del Ministerio, principalmente el decreto 22.439 por el cual se reglamenta el ejercicio de la odontología en todo el territorio de la república (Tabla 1). Todas las legislaciones analizadas han sido creadas hace varios años, no encontrándose hoy acorde a las necesidades de pacientes y profesionales de la salud. La realidad actual y los constantes avances científicos exponen la urgente necesidad de actualización de normativas legales existentes aplicadas al área de la salud, así como la creación de nuevas reglamentaciones que garanticen una atención médica y odontológica de calidad.

Tabla 1. Legislaciones y principales artículos analizados.

\begin{tabular}{|c|c|c|c|c|}
\hline & Constitución Nacional & Código Sanitario & Ley № 1.032/96 & Decreto 22.439 \\
\hline $\begin{array}{l}\text { Año de } \\
\text { promulgación }\end{array}$ & 1992 & 1980 & 1996 & 1998 \\
\hline $\begin{array}{l}\text { Objetivo } \\
\text { principal }\end{array}$ & $\begin{array}{l}\text { Asegurar la libertad, la } \\
\text { igualdad y la justicia. }\end{array}$ & $\begin{array}{l}\text { Regular las funciones del } \\
\text { Estado en el cuidado de la } \\
\text { salud y obligaciones de las } \\
\text { personas en la materia }\end{array}$ & $\begin{array}{l}\text { Distribuir de manera } \\
\text { equitativa y justa los recursos } \\
\text { nacionales en el Sector Salud }\end{array}$ & $\begin{array}{c}\text { Reglamentar el } \\
\text { ejercicio de la } \\
\text { odontología en el } \\
\text { Paraguay }\end{array}$ \\
\hline $\begin{array}{l}\text { Principales } \\
\text { artículos } \\
\text { analizados }\end{array}$ & Art. 68 - Art. 72 & Art. 1 - Art. 14 & $\begin{array}{l}\text { Art. } 1 \text { - Art. 22, } \\
\text { Art. 38- Art. } 39\end{array}$ & Art. 1 - Art. 8 \\
\hline
\end{tabular}

\section{DISCUSIÓN}

El Código Sanitario de la República del Paraguay ${ }^{6}$ establece que "se consideran profesiones en ciencias de la salud, todas aquellas disciplinas que tiendan, científica y técnicamente, al empleo de acciones integrales y coordinadas de promoción, protección, recuperación y rehabilitación del estado de bienestar físico, mental y social y al control de los factores condicionantes de 
la salud de las personas." Y que "para el ejercicio de los profesionales en ciencia de la salud se requiere contar con título expedido por las Universidades del país o revalidado por la Universidad Nacional de Asunción, que debe ser inscripto en el registro habilitado por el Ministerio"6.

Como ya fue citado anteriormente, el MSPBS es la más alta autoridad de salud y constituye el órgano encargado tanto de la reglamentación como de hacer cumplir las normativas existentes. Sin embargo, Paraguay no cuenta con mayor regulación del sector profesional de la salud. No existe una ley integral de sanidad ni regulaciones emitidas por los propios profesionales que sean obligatorias para los mismos (autorregulación de la profesión).

Comparando esta situación con la realidad odontológica brasilera, en 1964 fue promulgada la ley numero $4.324^{11}$, que instituye el Consejo Federal y los Consejos Regionales de Odontología. Cada uno de estos órganos tiene autarquía, es decir, está dotado de persona jurídica de Derecho Público y autonomía administrativa y financiera. Los mismos tienen por finalidad la supervisión de la ética profesional en toda la República del Brasil, y les corresponde velar y trabajar por el perfecto desempeño ético de la odontología, así como por el prestigio y buen concepto de la profesión y de quienes la ejercen legalmente ${ }^{11}$.

Por esta razón, estos consejos en el Brasil tienen atribuciones para fiscalizar y sancionar a los profesionales de la salud, a diferencia de lo que ocurre en Paraguay con el Circulo de Odontólogos de Paraguay (COP). El COP es una asociación gremial de colegas que velan por intereses comunes. Sin embargo, no posee atribuciones para fiscalización y sanción, debido a la ausencia, hasta hoy, de una ley que permita las colegiaturas profesionales.

La ley $5081^{12}$ de 24 de agosto de 1966 reglamenta el ejercicio de la odontología en el Brasil, y en forma similar, ocurre en Paraguay, donde el ejercicio de la profesión está regulado a través del Decreto $22.439^{8}$ de 1998. Ambas son legislaciones nacionales, es decir, con un nivel jerárquico superior $y$ alcance mayor a cualquier reglamentación emitida por organizaciones gremiales 0 autárquicas, que poseen atribuciones administrativas únicamente con representantes de la materia en cuestión a diferencia de las legislaciones nacionales que deben ser aprobados por representantes del pueblo.

Así, la Ley $5081^{12}$ y el Decreto $22.439^{8}$ tienen prelación sobre cualquier acto que sea emanado de organizaciones gremiales o autárquicas, que, deberán en todo momento actuar dentro del marco de la legislación vigente, no pudiendo emitir resoluciones que contradigan a lo dispuesto por las leyes y decretos. Aquí se incluye, a modo de ejemplo, la imposibilidad en Paraguay de que los consejos puedan sancionar a los profesionales.

$\mathrm{Si}$ bien no existe un enfoque regulatorio que sirva para todos los sistemas de salud $^{6}$ se entiende que la situación paraguaya actual, es decir, la ausencia de regulación durante un lapso de tiempo tan prolongado, ha provocado serias consecuencias en el sistema de salud nacional.

Una de las consecuencias de esta falta de regulación apropiada es el aumento 
de profesionales inconscientes y ajenos a la responsabilidad profesional. Profesionales publicando imágenes en redes sociales que revelan el secreto profesional, realizando procedimientos para los cuales no se encuentran habilitados o en ausencia de reglamentación adecuada para el efecto, poniendo en riesgo la salud pública y el bienestar de los ciudadanos ${ }^{13}$.

Teniendo en cuenta que los profesionales de la salud lidian diariamente con la vida de los individuos, y que cumplen un papel fundamental dentro del establecimiento y mantenimiento de la salud pública en general, se hace evidente no sólo la necesidad de actualizar las legislaciones para adaptarlas a los nuevos tiempos, sino la creación de nuevas reglamentaciones que puedan garantizar de forma apropiada un eficiente y adecuado ejercicio por parte de los profesionales de la salud en Paraguay $^{14-16}$.

La mayoría de sistemas de regulación de la profesión médica que a su vez han servido como base para regular los demás sectores de la salud, reconocen principalmente dos componentes fundamentales: por un lado, la regulación externa, entendida como las normas y regulaciones que establece el Estado gobierno en la materia, y por otro, la autorregulación que llevan a cabo los mismos profesionales del rubro, mediante sus códigos de ética y otras normativas de los colegios 0 consejos legalmente reconocidos ${ }^{17-20}$.

Particularmente en Paraguay, existe un gran problema, que es la ausencia hasta hoy de una ley que habilite y regule las colegiaturas profesionales. Realizando una búsqueda en la literatura local, es posible encontrar varios artículos de diferentes rubros reclamando esta situación. Químicos $^{21}$, abogados ${ }^{22}$, ingenieros ${ }^{23}$, resaltan la necesidad de una correcta fiscalización. Las falencias que atraviesan los profesionales en Paraguay son consecuencia de las legislaciones poco claras establecidas para la profesión, afirman ${ }^{21}$.

La colegiación profesional obligatoria es un tema bastante polémico, y normalmente se alega que la Constitución Nacional prohíbe la Colegiación obligatoria. Esta justificativa ha sido ampliamente utilizada por aquellos que rechazan el control de ejercicio profesional y el funcionamiento de Tribunales de Conducta que apliquen un Código de Ética en las diferentes profesiones ${ }^{24}$. Esta discusión ha sido ampliamente abordada e incluso superada en la mayoría de los países, pero sin lugar a duda es un tema complejo que debería ser explorado con mayor detenimiento ${ }^{25}$.

Otro factor a considerar es el aumento excesivo de universidades en Paraguay $^{26}$, facilitando así el acceso a la educación universitaria y por consiguiente formando anualmente una gran cantidad de profesionales en los diferentes rubros. Hasta el año 2003 en Paraguay había apenas 23 universidades habilitadas, mientras que en 2017 aumentó a 54 (públicas y privadas), sin embargo, 31 de ellas no posee una carrera acreditada ${ }^{27}$.

El exceso de profesionales sin control en el mercado ${ }^{26}$ resulta en competencia desleal, publicidad engañosa, precios que desvalorizan la profesión, 
trabajo deshumanizado y con fallas técnicas, etc., reflejando nuevamente la necesidad de reglamentación y fiscalización de las profesiones ${ }^{28-31}$.

Esta situación aplicada al área de la salud, donde una falla técnica puede resultar en secuelas e inclusive en la muerte de un individuo ${ }^{32-33}$, es sumamente alarmante y se convierte en una necesidad de salud pública. La falta de tiempo para un correcto diagnóstico debido al número mínimo de consultas que un médico u odontólogo debe realizar en una mañana por políticas internas de establecimientos de salud, o por la utilización de materiales más económicos para justificar los costos, es un precio altísimo, que la población no debería pagar $^{34}$. Todos los ciudadanos tienen derecho a una atención médica y odontológica de calidad, ejercida por profesionales capacitados.

Expuesto esto, es posible entender que, actualmente en Paraguay, resulta inútil discutir acerca de los límites de actuación de odontólogos y médicos, o sobre la legitimidad de la realización de procedimientos faciales exclusivamente estéticos por profesionales odontólogos, un tema muy polémico en la actualidad y ampliamente discutido por colegas pertenecientes a ambos gremios, cuando en realidad la solución real es la creación de legislaciones que reglamenten el ejercicio profesional, delimitando áreas de actuación, atribuciones, prohibiciones y penalidades. $Y$ por supuesto, la aprobación de una ley adecuada de la colegiación profesional, otorgándoles a los diferentes colegiados, atribuciones tanto para la fiscalización como la penalización de aquellos profesionales que se encuentren infringiendo normativas legales y éticas, a fin de salvaguardar la salud de todos los ciudadanos.

\section{CONCLUSIÓN}

Fue posible exponer la falta de regulación adecuada en el área de la salud en Paraguay, y las consecuencias negativas de esta situación, así como a concluir que la creación de un marco regulatorio actualizado y moderno es una necesidad de salud pública que promoverá los intereses de la sociedad al favorecer y facilitar la prestación de servicios de atención médica de alta calidad.

\begin{abstract}
One of the State's main functions is to safeguard the health of its citizens. In Paraguay, this role is performed by the Ministry of Public Health and Social Welfare, which is the competent authority for the provision, regulation and supervision of all health services in the country. The majority of health care regulations were introduced years ago and are currently out of date. Medical errors can and often lead to death. This is why modernizing and updating regulations of professional practice in the health care sector is a priority and an urgent public health necessity. The purpose of this article was to reveal the existing problems caused by the lack of adequate health related regulations, as well as to establish that said regulation constitutes a public health necessity. First, comprehensive research of existing local legislations regarding professional practice was made. Next, each regulation was thoroughly analyzed, followed by a discussion about the applicability as well as the limitations of each regulation to each specific situation. Through this work, it was possible to uncover the lack of adequate regulation in the health care sector in Paraguay, and the negative consequences of this scenario, as well as to arrive to the conclusion that the creation of an updated and modern regulatory framework is an urgent public necessity that will advance the interests of society by favoring and encouraging high-quality health care services.
\end{abstract}

\title{
KEYWORDS
}

Forensic dentistry; Professional practice; Public health; Regulation. 


\section{REFERENCIAS}

1. Irazusta MA. Responsabilidad profesional. En Barragán HR. Moiso A, Mestorino MA, Ojeda OA. Fundamentos de salud pública. La Plata; 2007 p. 615-8. Disponible en: http://sedici.unlp.edu.ar/bitstream/handle/109 15/29128/Documento completo .pdf?seque nce $=4$.

2. Colegio Profesional de maestros mayores de obras y técnicos de Entre Ríos. El concepto de ejercicio profesional. Entre Ríos. [Internet]; [consultado el 19 de febrero de 2018] Disponible en: http://www.copmmoter.org.ar/textocomp.asp?i $\underline{d=161}$.

3. Gobierno Nacional. Ministerio de Salud pública y bienestar social. Legislación en Salud [Internet]; [consultado el 17 de febrero de 2018] Disponible en: http://www.mspbs.gov.py/legislacion-ensalud/.

4. Gobierno Nacional. Ministerio de Salud pública y bienestar social. Informe final, ejercicio fiscal. Asunción, 2010. [Internet]; [consultado el 20 de febrero de 2018] Disponible en:

http://www.mspbs.gov.py/legislacion-ensalud/.

5. Gobierno Nacional. Constitución Nacional de 20 de junio de 1992. [consultado el 25 de febrero de 2018] Disponible en: http://jme.gov.py/transito/leyes/1992.html.

6. Gobierno Nacional. Ministerio de Salud pública y bienestar social. Ley 836 del código sanitario. [Internet]; [consultado el 17 de febrero de 2018] Disponible en: www.who.int/fctc/reporting/Paraguay annex8 ?health act.pdf.

7. Gobierno Nacional. Ley 1032 de 1996. Que crea el sistema Nacional de Salud. [consultado el 14 de setiembre de 2018] Disponible

en: http://www.vertic.org/media/National\%20Legis lation/Paraguay/PY Ley Sistema Nacional de Salud.pdf.

8. Gobierno Nacional. Decreto 22.439 de 14 de agosto de 1998. Por el cual se reglamenta el ejercicio de la odontología en todo el territorio de la República. [consultado el 20 de febrero de 2018] Disponible en: http://www.mic.gov.py/mic/site/comercio/dgcs/ pdf/7 ServiciosProfesionales/Decreto22439.p df.

9. Perea RSS. La calidad en el desarrollo profesional: avances y desafíos. Educ Med Super, 2000;14(2):136-47. Disponible en: http://scielo.sld.cu/scielo.php?script=sci artte xt\&pid=S0864-21412000000200003\&lng=es.

10. Borracci RA, Mauro VM. El profesionalismo médico, los modelos de regulación y la autonomía profesional. Rev Argent Cardiol, 2015;83(3):241-3 .

http://dx.doi.org/10.7775/rac.es.v83.i3.5834

11. Brasil. Lei n. 4.324 de 14 de abril de 1964. Institui o Conselho Federal e os Conselhos Regionais de Odontologia, e dá outras providências. [consultado el 14 de setiembre de 2018] Disponible en: http://www.planalto.gov.br/ccivil 03/LEIS/195 0-1969/L4324.htm

12. Brasil. Lei n. 5081 de 24 de agosto de 1966. Regula 0 excercício da odontologia. [consultado el 14 de setiembre de 2018] Disponible en: http://www.planalto.gov.br/ccivil 03/leis/L508 1.htm.

13. Brinkerhoff DW. Accountability and health systems: toward conceptual clarity and policy relevance. Health Policy and Planning, 2004;19(6):371-9. Disponible en: https://academic.oup.com/heapol/articleabstract/19/6/371/579576.

14. Frenk J, Chen L, Bhuttad ZA, Cohene J, Crispf $\mathrm{N}$, Evans $\mathrm{T}$, et al. Profesionales de la salud para el nuevo siglo: transformando la educación para fortalecer los sistemas de salud. Educ Med. 2015; 16(1):9-16. http://dx.doi.opy/10.1016/j.edumed.2015.04.01 1.

15. González JB. Del Pozo Serrano FJ. Health education adolescents and approach from social pedagogy in contexts of vulnerability. Salus Uninorte, 2017;33(2):213-23. Disponible en:

http://www.scielo.org.co/scielo.php?script=sci abstract\&pid=S0120-55522017000200213.

16. Yahya S, Sheikh AS. Meeting the current medical education challenges in resource Poor settings. Pak J Med Sci 2012; 28(4). Disponible en: http://pjms.com.pk/index.php/pjms/article/dow nload/2457/614.

17. Argentina. Presidencia de la Nación. Ministerio de Salud. Regulación del ejercicio profesional. Argentina. [Internet]; [consultado el 18 de febrero de 2018] Disponible en: http://www.msal.gob.ar/observatorio/index.ph p/fuerza-de-trabajo/regulacion-del-ejercicioprofesional.

18. Mera Jorge et al. La certificación de los profesionales médicos en la Argentina. 
Comisión Nacional Salud Investiga, 2007, p 23. [Internet]; [consultado el 18 de febrero de 2018] Disponible en: http://www.msal.gob.ar/observatorio/images/s tories/documentos fuerza trabajo/FT Catego ria Regulacion/MERA ETAL Lacertificacinde profesioanalesmedicosenargentina.pdf.

19. Pino F. Ley de ejercicio profesional su necesidad. [Internet]; [consultado el 18 de febrero de 2018] Disponible en: http://www.cpi.org.py/doc/biblioteca/articulo I ey ejercicio profesional ve.doc.

20. Nigenda G, Machado MH. Modelos de regulación profesional de los médicos en América Latina: elementos teóricos para su análisis. Cad. Saúde Públ. 1997; 13(4):68592. Disponible en: www.scielo.br/pdf/csp/v13n4/0152.pdf.

21. Schwarz. R. Químicos del Paraguay anhelan colegiaturas. ABC Digital. Asunción, Paraguay, 2012. Disponible en: http://www.abc.com.py/edicionimpresa/locales/quimicos-del-paraguayanhelan--una-colegiatura-398767.html.

22. Camacho E. Ley de las colegiaturas es constitucional. ABC Digital. Asunción, Paraguay, 2014. Disponible en: http://www.paraguay.com/nacionales/ley-decolegiatura-es-constitucional-segun-emiliocamacho-109188.

23. Centro paraguayo de ingenieros [website] Disponible en: http://www.cpi.org.py/cepup.html

24. Murcia JG. Derecho de sindicación y colegios profesionales en la jurisprudencia constitucional. Rev Esp de Der Constit. 1991; 31:151-198. Disponible en: http://www.jstor.org/stable/24879732.

25. Guier F. Memoria presentada por la sociedad interamericana de prensa, como amicus curiae, en la solicitud de opinión consultiva formulada por el gobierno de costa rica ante la corte interamericana de derechos humanos respecto de la compatibilidad de la ley costarricense $n$ o 4420, que establece la colegiatura obligatoria de periodistas, con la convención americana sobre derechos humanos. Revista Chilena de Derecho, 1985; 12(3):457-475. Disponible en: http://www.jstor.org/stable/41608231.

26. Rivarola D. La universidad paraguaya hoy. Rev. Aval educ super. Campinas, 2008; 13(2):533-578. Disponible en: http://www.scielo.br/pdf/aval/v13n2/14.pdf.

27. Gobierno nacional. Nomina de universidades del sector oficial y privado con macro legal de aprobación para su funcionamiento. Disponible http://www.aneaes.gov.py/aneaes/index.php/c t-menu-item-31.

28. Flore BB, Montes KL. La situación del mercado laboral de profesionistas. Rev. Educ sup. México, 2016;39(156). Disponible en: http://www.scielo.org.mx/scielo.php?script=sci arttext\&pid=S0185-27602010000400002.

29. Carrera J. La guerra de los dentistas. [blog]. Barcelona, 2014. Disponible en: http://cadenaser.com/programa/2014/12/10/h oy por hoy/1418207619 156810.html.

30. Ferreira NP, Ferrerira AP, Freire MCM. Mercado de trabalho na odontología: contextualização e perspectivas. Rev Odontol UNESP. 2013; 42(4): 304-309. Disponible en: http://www.scielo.br/pdf/rounesp/v42n4/11.pdf

31. Paranhos LR, Ramos AL, Scanavini MA, Ricci ID. Análise do mercado de trabalho odontológico na região Sudeste do Brasil. Rev Assoc Paul Cir Dent. 2009;63(1):57-63.

32. Paraguay.com. Denuncia a odontóloga por mala práxis tras extracción sin anestesia. Paraguay, 2018. [consultado el 14 de setiembre de 2018] Disponible en: http://www.paraguay.com/nacionales/denunci a-a-odontologa-por-mala-praxis-trasextraccion-sin-anestesia-173080.

33. Oviedo S, Gutierrez AC. "Cada vez tenemos mas casos", die el doctor Pablo Lemir, diretor de Medicina Legal y Forense del Ministerio Público. 78 juntas médicas ser realizaron entre el último semestre del año 2009 y el 2010 ante casos de mala praxis. Ultima hora, 2009. [consultado el 14 de setiembre de 2018] Disponible en: https://www.ultimahora.com/desde-2009-seprodujeron-78-pericias-negligencia-medican433613.html.

34. Oliveira FT. Ética profissional odontológica: análise do conhecimento de discentes e cirurgiões-dentistas sobre o código deontológico da profissão. [dissertação]. Programa de pós- graduação em ortodontia e odontologia em saúde coletiva. Baurú, 2006. Disponible en: www.teses.usp.br/teses/disponiveis/25/.../Fer nandoOliveira.pdf. 